\title{
AN ILLUSTRATION OF THE DUALITY TECHNIQUE IN SEMI-CONTINUOUS LINEAR PROGRAMMING*
}

\author{
F. De VyLder
}

\begin{abstract}
We give a complete parametric solution of the following problem: Find a claim size distribution $F$ on the finite interval $[o, \omega]$, maximizing the stop-loss premium corresponding to a given excess $e$, under the constraints that the furst moment of $F$ be at most equal to $\mu$ and the second at most equal to $\nu$ The method used is the duality technique in semi-continuous linear programming described in DE VYLDER (1978) This technique is summarized, without proofs, in the first part of the paper.
\end{abstract}

\section{THE DUALITY TECHNIQUE IN SEMI-CONTINUOUS LINEAR PROGRAMMING}

\subsection{Generalnzed Matruces}

Let $A_{i}^{x}$ be defined for $i \in I=\{1,2, \ldots, m\}$ and $x \in K \subseteq R$. In most situations $K$ is a finite interval, but in many considerations it might be a rather general set in $R$. We consider $A$ as a generalized matrix, with the set $I$ of row-indices and the set $K$ of column-indices. The augmented matrix $A .=(A, 1)$ is formed by the matrix $A$ followed by the unit matrix 1 of dimension $m$. We suppose that the columns of this unit matrix are indexed by any indices $\sigma_{1}, \sigma_{2}, \ldots, \sigma_{m}$ (not necessarly numbers), of course none in $K$. These indices are called slack indices. Let we denote by $S$ the set of slack indices. If $M=\left(x_{1}, x_{2}, \ldots, x_{r}\right)$ is any finite sequence of elements in $K+S$, we denote by $A^{M}$ the usual matrix with columns $A^{x_{1}}, A^{x_{2}}, \ldots, A_{r}^{x_{r}}$, to be defined in a moment. If $x_{j}=x \in K$, then $A^{x}$. 1s the column $\left(A_{1}^{x}, A_{2}^{x}, \ldots, A_{m}^{x}\right)^{\prime}$. If $x_{j}$ is a slack index, say $x_{j}=\sigma_{k}$ $(1 \leqslant k \leqslant m)$, then the column $A^{\sigma_{k}}$ is the column with $m$ elements $(0,0, \ldots$, $0,1,0, \ldots, 0,0)$, where the 1 is at the $k$-th place.

Similarly, if $b^{x}$ is defined for $x \in K$, we consider $b$ as a generalized row with the set $K$ as column-indices. The augmented row $b .=(b, 0)$ is formed by $b$ followed by $m$ zero's. The usual row $b^{M}$. is the row

$$
b^{M}=\left(b^{x_{1}}, b^{x_{2}}, \ldots, b^{x_{1}}\right),
$$

where $b^{x_{j}}=0$ if $x_{j}$ is a slack index.

In the sequel, the sequence $M$ will have exactly $m$ elements. Thus $A^{M}$. will be a square matrix. The order of the elements in $M$ is irrelevant, but usually we shall suppose that the indices in $K$ are written first, in the natural order, followed by the slack indices in the order induced by their subscripts.

* Presented at the 14th ASTIN Colloquium, Taormina, October 1978. 


\subsection{Problems in Semi-Continuous Linear Programming}

In the sequel $K$ is always fixed. $F$ is an unknown distribution function on $R$ (not necessarily a probability distribution function: the total mass induced by $F$ on $R$ may be different from 1, but it must be finite, for simplicity). In fact, the mass induced by $F$ on $R-K$ is completely irrelevant, since all integrals considered in the sequel will be taken over $K$ (then this set is generally not indicated) or over a subset of $K$, relatively to the distribution function $F$.

Let $A, b$ be defined as in 1.1. and let a be the column $a=\left(a_{1}, a_{2}, \ldots, a_{m}\right)^{\prime}$. Then the problem noted $(A, a, b, F, M A X)$ is to find a distribution function $F$ maximizing $\int b^{x} d F_{x}$ under the constraints $\int A_{i}^{x} d F_{x} \leqslant a_{i}(i \in I)$. The dual problem is to find a row $y=\left(y^{1}, y^{2}, \ldots, y^{m}\right)$ minimizing $y a=\Sigma y^{i} a_{i}$ under the constraints $y^{i} \geq 0(i \in I), \sum_{i} y^{i} A_{i}^{x} \geq b^{x}(x \in K)$.

\subsection{A Fundamental Theorem}

If $F$ satisfies the constraints of the problem $(A, a, b, F, M A X)$, if $y$ satisfies the constraints of the dual problem and if

$$
\int b^{x} d F_{x}=\Sigma y^{i} a_{i},
$$

then $F, y$ are solutions of the problem and its dual respectively.

\subsection{The Duality Technique}

Various tests have proved that the following technique for solving the problem $(A, a, b, F, M A X)$ works in rather general circumstances, provided step 1 can be executed. It would be highly interesting to know the most general conditions on $K, A, a, b$ for the technique to succeed.

STEP 1. Find a solution $y$ of the dual problem.

STEP 2. Find $M=\left(x_{1}, x_{2}, \ldots, x_{m}\right)$ satisfying $y A^{M}=b^{M}$. The indices $x_{j}$ (slack or not) can be searched for the one after the other. Each $x$ satisfying y $A^{x}=b^{x}$ may be an element of the sequence $M$. In view of the following step try to find as many different indices as possible. Ideal, but not always realizable (nor necessary) is that $A^{M}$. be inversible.

STEP 3. Find a column $z=\left(z_{1}, z_{2}, \ldots, z_{m}\right)^{\prime}$ satisfying $A^{M} z=a, z_{i} \geq 0$ $(i \in I)$. Then define the discrete distribution $F$ in the following way. For each $x_{j}$ in $M, x_{j} \in K$ (thus $x_{j}$ not a slack index), place the mass $z_{j}$ at the point $x_{j}$. If e.g. $x_{1}=x_{2}$, then the mass $z_{1}+z_{2}$ must be placed at $x_{1}=x_{2}$ and similarly if more indices are equal.

STEP 4. Verify (1). Then, by the fundamental theorem, it follows that $F$ and $y$ are solutions (for $y$ this is in fact a confirmation) since the relations $A^{M} z=a, z_{i} \geq 0(i \in I)$ imply that $F$ satisfies the constraints of the given problem. 


\section{THE MAIN PROBLEM CONSIDERED IN THIS PAPER}

\subsection{Original Problem}

Find a claim size probability distribution $F$ on $K=[0, \omega]$ maximizing the stop-loss premium

$$
\int_{0}^{\infty}(x-e) d F_{x}
$$

corresponding to the excess $e$, under the constraints

$$
\int x d F_{x} \leqslant \mu, \int x^{2} d F_{x} \leqslant \nu .
$$

\subsection{Equivalent Problem}

Find a distribution $F$ on $K$ maximizing (2) under the constraints

$$
\int d F_{x} \leqslant 1, \int x d F_{x} \leqslant \mu, \int x^{2} d F_{x} \leqslant \nu .
$$

That this problem is equivalent results from the fact that the first members in the relations (3) do not depend on the probability mass at the origin of $K$. If we have a distribution $F$, solution of the second problem, we can make a probability distribution of it by placing, if necessary, the missing probability at the origin. (It will turn out that this modification will not even be necessary.)

Thus, our main problem is the problem

$$
(A, a, b, F, M A X) \text {, where } I=\{1,2,3\}, K=[0, \omega]
$$

$$
\left(\begin{array}{l}
A_{1}^{x} \\
A_{2}^{x} \\
A_{3}^{x}
\end{array}\right)=\left(\begin{array}{l}
1 \\
x \\
x^{2}
\end{array}\right)(x \in K), a=\left(\begin{array}{l}
1 \\
\mu \\
\nu
\end{array}\right), b^{x}=\left\{\begin{array}{c}
0 \text { for } 0 \leqslant x<e \\
x-e \text { for } e \leqslant x \leqslant \omega
\end{array}\right.
$$

\subsection{Dual Problem}

The dual problem is to find $y=(u, v, w)$ minimizing $u+v \mu+w v$ under the constraints

$$
u \geqslant 0, v \geqslant 0, w \geqslant 0, \quad u+v x+w x^{2} \geqslant b x(0 \leqslant x \leqslant \omega) .
$$

Of course, these constraints are equivalent to

$$
u \geqslant 0, v \geqslant 0, w \geqslant 0, \quad u+v x+w x^{2} \geqslant x-e(e \leqslant x \leqslant \omega) .
$$

\subsection{The Parameters of the Problem}

The problem is one with four parameters $\omega, e, \mu, \nu$ and we shall give its solution whatever be the relations among these parameters. For the sake of completeness, unrealistic cases have been included. Evidently, we always assume

(9)

$$
\mu>0, \quad \nu>0, \quad 0 \leqslant e<\omega
$$


and also, until section 5 , the relations

$$
\mu<\sqrt{\nu}, \quad \nu<\mu \omega
$$

implying

$$
\nu<\omega^{2}, \quad \mu<\omega
$$

Suppose for a moment that we have not the first relation (10), i.e. that we have $\sqrt{\nu} \leqslant \mu$. Then it follows from the last relation (4) that

$$
\int x d F_{x} \leqslant\left[\int x^{2} d F_{x}\right]^{1 / 2} \leqslant \sqrt{\nu} \leqslant \mu
$$

and the second constraint (4) becomes superfluous. Thus, the case $\sqrt{\nu} \leqslant \mu$ leads to a simpler problem that shall be considered separately. Note that the first relation (12), well-known when $F$ is a probability distribution, is also valid if $F$ is defective.

Similarly, suppose for a moment that we have not the last relation (10), i.e. that we have $\mu \omega \leqslant v$. Then it follows from the second relation (4) that

$$
\int x^{2} d F_{x} \leqslant \omega \int x d F_{x} \leqslant \omega \mu \leqslant \nu
$$

and then the last constraint (4) is superfluous. Again we are faced with a simpler problem, in fact a problem equivalent to the Gagliardi-Straub (1974) problem.

\section{STEP 1. SOLUTION OF THE DUAL PROBLEM}

For each $x(e \leqslant x \leqslant \omega)$, considered as parameter, we imagine that we represent the part of the plane $u+v x+w x^{2}=x-e$ situated in the positive octant of the $(u, v, w)$ space (fig. 1$)$.

Let

$$
P_{x}=(x-e, 0,0), Q_{x}=\left(0, \frac{x-e}{x}, 0\right), R_{x}=\left(0,0, \frac{x-e}{x^{2}}\right)
$$

be the intersection with the $u, v, w$-axis respectively.

When $x$ increases from $e$ to $\omega, P_{x}$ and $Q_{x}$ remove monotonously from the origin. For $R_{x}$, two cases must be considered:

Case $C_{1}: 2 e \geqslant \omega$. Then $R_{x}$ goes up from the height o to the height $\frac{\omega-e}{\omega^{2}}$.

Case $C_{2}: 2 e<\omega$. Then $R_{x}$ goes up from the height o to the height $1 / 4 e$ (for $x=2 e$ ), then comes down to the height $\frac{\omega-e}{\omega^{2}}$. 


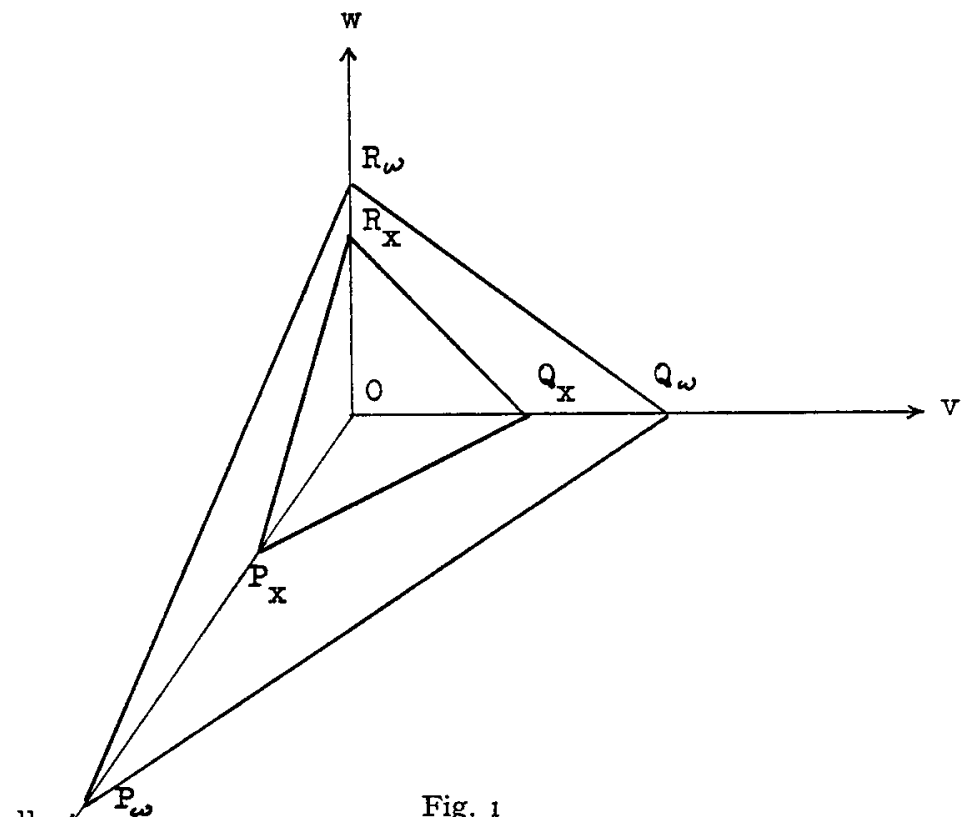

Fig. 1

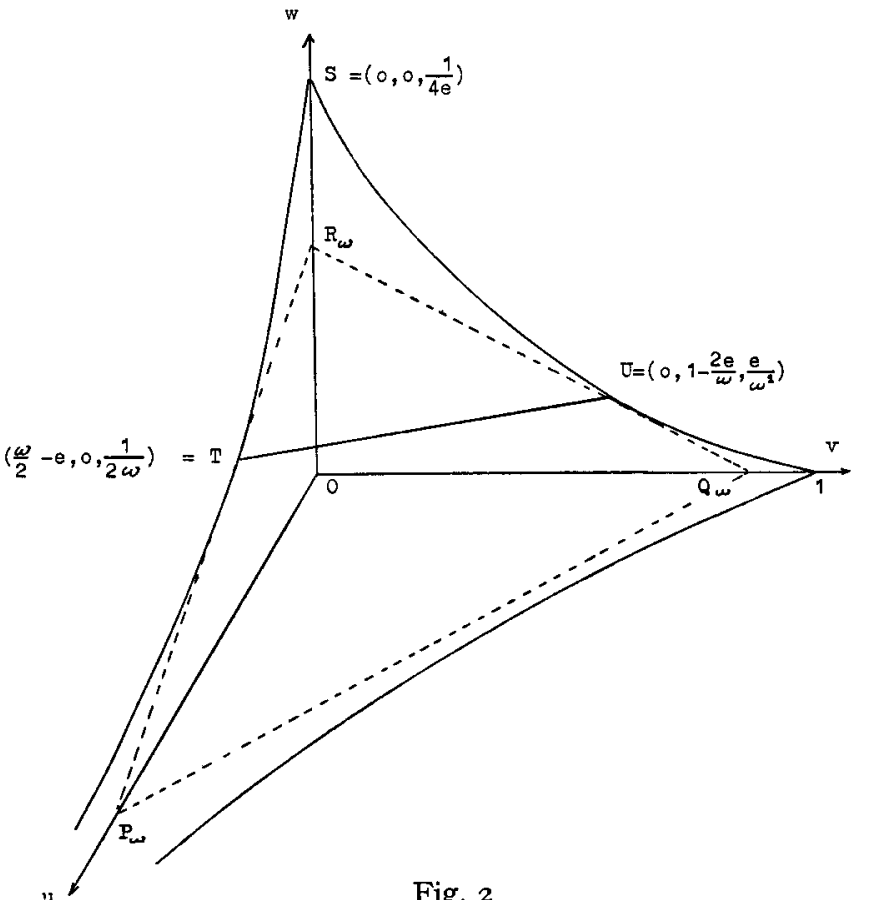

Fig. 2 


\subsection{Study of Case $C_{1}$}

It is clear, in the case $C_{1}$, that the minimum of $u+v \mu+v v$, subject to the given constraints, is obtained for $(u, v, w)$ in the triangle $P_{\omega} Q_{\omega} R_{\omega}$. For reasons of linearity it must be obtained at one of the corners. From (10), (11) it is immediate that the minimum is obtained at $R_{\omega}$. Thus we have the solution and corresponding minimum:

$$
u=0, v=0, w=\frac{\omega-e}{\omega^{2}}, M I N=(\omega-e) \frac{\nu}{\omega^{2}} .
$$

\subsection{Study of Case $C_{2}$}

Since $R_{x}$ goes up and down when $x$ increases from $e$ to $\omega$, the triangles $P_{x} Q_{x} R_{x}$ have a superior envelope. We can find its equation by eliminating $x$ between the relation $u+v x+w x^{2}=x-e$ and its derivative in $x$. The equation of the envelope is

$$
w=\frac{1}{4} \frac{(1-v)^{2}}{(u+e)} .
$$

Of course, only the part in the positive octant must be used, and even not completely, because we have the restriction $x \leqslant \omega$. It is calculated that the triangle $P_{\omega} Q_{\omega} R_{\omega}$, corresponding to the maximum value of $x$, is tangent to the envelope along the straight segment $T U$ (fig. 2), where

$$
T=\left(\frac{\omega}{2}-e, 0, \frac{1}{2 \omega}\right), U=\left(0,1-\frac{2 e}{\omega} \frac{e}{\omega^{2}}\right) .
$$

Then the minimum of $u+v \mu+w v$, under the given constraints, is obtained for $(u, v, w)$ in the part $S T U$ of the envelope, or for $(u, v, w)$ in the plane portion $P_{\omega} Q_{\omega} U T$.

In order to study the variation of $u+v \mu+w v$ on $S T U$, let we take $u$ and $v$ as independent variables and let we use (14). Then the quantity to be minimized equals, on $S T U$ :

$$
f(u, v)=u+v \mu+\frac{\nu}{4} \frac{(1-v)^{2}}{(u+e)} .
$$

It is easily calculated that there can be no $u, v$ annulating the partial derivatives $f_{u}^{\prime}, f_{v}^{\prime}$. We conclude that there can be no minimum at the interior of $S T U$. Therefore, and also for reasons of linearity, the minimum of $u+v \mu+w v$ is only possible for $(u, v, w)$ on $S T$, on $S U$, at $P_{\omega}$, at $Q_{\omega}$.

\subsubsection{Study of the Variation of $f(u, v)$ along $S T$}

We have, for $S T$ :

$$
g(u)=f(u, 0)=u+\frac{v}{4} \frac{1}{u+e},\left(0 \leqslant u \leqslant \frac{\omega}{2}-e\right),
$$




$$
\begin{aligned}
& g^{\prime}(u)=1-\frac{\nu}{4} \frac{1}{(u+e)^{2}}, g^{\prime \prime}(u) \geqslant 0, \\
& g^{\prime}(u)=\text { o for } u=\frac{1}{2} \sqrt{v}-e .
\end{aligned}
$$

Therefore,

if $\frac{1}{2} \sqrt{\nu}-e \leqslant 0$, then: Minimum on $S T=$ Value at $S=\frac{\nu}{4 e}$.

if $0<\frac{1}{2} \sqrt{\nu}-e$, then: Minimum on $S T=$ Value at $\left(\frac{1}{2} \sqrt{\nu}-e, 0, \frac{1}{2 \sqrt{\nu}}\right)$

$$
=\sqrt{\nu}-e \text {, because } \frac{1}{2} \sqrt{\nu}-e<\frac{\omega}{2}-e \text { by }(11) \text {. }
$$

\subsubsection{Study of the Variation of $f(u, v)$ along $S U$}

We have, for $S U$ :

$$
\begin{aligned}
& h(v)=f(0, v)=v \mu+\frac{\nu}{4 e}(1-v)^{2},\left(0 \leqslant v \leqslant 1-\frac{2 e}{\omega}\right), \\
& h^{\prime}(v)=\mu-\frac{\nu}{2 e}(1-v), h^{\prime \prime}(v) \geqslant 0, \\
& h^{\prime}(v)=\text { o for } v=1-2 \frac{e \mu}{\nu} .
\end{aligned}
$$

Therefore,

if $1-2 \frac{e \mu}{\nu} \leqslant 0$, then: Minimum on $S U=$ Value at $S=\frac{\nu}{4 e}$.

if $0<1-2 \frac{e \mu}{\nu}$, then: Minimum on $S U=$ Value at $\left(0,1-2 \frac{e \mu}{\nu}, \frac{e \mu^{2}}{\nu^{2}}\right)$

$$
=\mu\left(1-\frac{e \mu}{\nu}\right) \text {, because } 1-2 \frac{e \mu}{\nu}<1-2 \frac{e}{\omega} \text { by }(10) \text {. }
$$

3.2.3. We note that the value of $u+v \mu+w \nu$ at $P_{\omega}=(\omega-e, 0,0)$ is $(\omega-e)$ and at $Q_{\omega}=\left(0, \omega-\frac{e}{\omega}, 0\right)$ it is $(\omega-e) \frac{\mu}{\omega}$.

\subsection{Subclassification of Case $C_{2}$}

The subcases of $C_{2}$ indicated in table 1 , with, in the right column the possible minimum, result from the discussion in 3.2.1 and 3.2.2. Note that, since $\mu / \omega<1$, the value $(\omega-e)$ at $P_{\omega}$ can already be abandoned against the value $(\omega-e) \mu / \omega$ at $Q_{\omega}$. 
TABLE I

\begin{tabular}{ll}
\hline \hline Subcases of $C_{2}(2 e<\omega)$ & Possible minimum \\
\hline$C_{21}: \nu \leq 4 e^{2}, \nu \leq 2 e \mu$ & $\frac{\nu}{4 e},(\omega-e) \frac{\mu}{\omega}$ \\
$C_{22}: \nu \leq 4 e^{2}, \nu>2 e \mu$ & $\frac{\nu}{4 e}, \mu\left(1-\frac{e \mu}{\nu}\right),(\omega-e) \frac{\mu}{\omega}$ \\
$C_{23}: \nu>4 e^{2}, \nu \leq 2 e \mu$ & Irrelevant (see discussion) \\
$C_{24}: \nu>4 e^{2}, \nu>2 e \mu$ & $\sqrt{\nu}-e, \mu\left(1-\frac{e \mu}{\nu}\right),(\omega-e) \frac{\mu}{\omega}$ \\
\hline
\end{tabular}

The first relation (10) and the relation $\nu \leqslant 2 e \mu$ appearing twice in table 1 , imply $\nu=2 e \sqrt{v}, \nu \leqslant 4 e^{2}$. This means that case $C_{23}$ is impossible and that in case $C_{21}$, the condition $\nu \leqslant 4 e^{2}$ is superfluous. In case $C_{21}$, we can abandon the potential minimum $(\omega-e) \frac{\mu}{\omega}$, because, by adding the relations $\frac{\nu}{4 e} \leqslant \frac{\mu}{2}, \frac{\mu e}{\omega}<\frac{\mu}{2}$, we have $\frac{\nu}{4 e}<(\omega-e) \frac{\mu}{\omega}$. The relation $\mu\left(1-\frac{e \mu}{\nu}\right)<(\omega-e) \frac{\mu}{\omega}$ is seen to be equivalent to $\nu<\mu \omega$. Therefore, by $(10),(\omega-e) \frac{\mu}{\omega}$ can also be abandoned in $C_{22}$ and $C_{24}$. From o $\leqslant(2 e \mu-\nu)^{2}$ results that $\frac{\nu}{4 e}$ can be abandoned in $C_{22}$. Finally, in case $C_{24}$, let we add the relations $\frac{e}{\sqrt{v}}<\frac{1}{2}, \frac{e \mu}{\nu}<\frac{1}{2}$. We obtain $\frac{e}{\nu}(\sqrt{\nu}+\mu)<1$. After multiplication by $\sqrt{\nu}-\mu>0$, we have a relation equivalent to $\mu\left(1-\frac{e \mu}{\nu}\right)<\sqrt{\nu}-e$, showing that $\sqrt{\nu}-e$ can be abandoned. Then $C_{22}$ and $C_{24}$ can be amalgamated in one case, say $C^{\prime}{ }_{22}$, defined by $\nu>2 e \mu, 2 e<\omega$.

\subsection{Final Table}

The preceding discussion is resumed in table 2 .

TABLE 2

\begin{tabular}{lll}
\hline Cases & $\begin{array}{l}\text { Point }(u, v, w) \\
\text { giving the } \\
\text { minimum }\end{array}$ & MIN \\
\hline$C_{1}: 2 e \geq \omega$ & $\left(0,0, \frac{\omega-e}{\omega^{2}}\right)$ & $(\omega-e) \frac{\nu}{\omega^{2}}$ \\
$C_{21}: 2 e<\omega, \nu \leq 2 e \mu$ & $\left(0,0, \frac{1}{4 e}\right)$ & $\frac{\nu}{4 e}$ \\
$C^{\prime}{ }_{22}: 2 e<\omega, \nu>2 e \mu$ & $\left(0,1-2 \frac{e \mu}{\nu}, \frac{e \mu^{2}}{\nu^{2}}\right)$ & $\mu\left(1-\frac{e \mu}{\nu}\right)$ \\
\hline
\end{tabular}




\section{FURTHER STEPS}

\subsection{Case $C_{1}$}

STEP 2.

We look for $x \in K$ satisfying

$$
\left(0,0, \frac{\omega-e}{\omega^{2}}\right)\left(\begin{array}{c}
1 \\
x \\
x^{2}
\end{array}\right)= \begin{cases}0 & \text { if } x<e \\
x-e & \text { if } x \geqslant e\end{cases}
$$

The solutions are $x=0$ and $x=\omega$. Since

$$
\left(0,0, \frac{\omega-e}{\omega^{2}}\right)\left(\begin{array}{l}
0 \\
1 \\
0
\end{array}\right)=0,
$$

the slack index $\sigma_{2}$ can also be used. Then $M=\left(0, \omega, \sigma_{2}\right)$.

\section{STEP 3.}

We look for $z_{1}, z_{2}, \xi$ (the last is a slack variable corresponding to $\sigma_{2}$ ) satisfying

$$
\left(\begin{array}{ccc}
1 & 1 & 0 \\
0 & \omega & 1 \\
0 & \omega^{2} & 0
\end{array}\right) \quad\left(\begin{array}{l}
z_{1} \\
z_{2} \\
\xi
\end{array}\right)=\left(\begin{array}{l}
1 \\
\mu \\
\nu
\end{array}\right) .
$$

The solution is $z_{1}=1-\frac{\nu}{\omega^{2}}, z_{2}=\frac{\nu}{\omega^{2}}, \xi=\mu-\frac{\nu}{\omega}$.

Thus, $F$ has two atoms, one at $o$, the other at $\omega$, with respective masses

$$
m_{0}=1-\frac{\nu}{\omega^{2}}, m_{\omega}=\frac{\nu}{\omega^{2}} \text {. }
$$

STEP 4 .

$$
\int_{e}^{\omega}(x-e) d F_{x}=(\omega-e) \frac{\nu}{\omega^{2}}=\text { Value MIN in table } 2 .
$$

\subsection{Case $C_{21}$}

STEP 2.

We look for $x \in K$ satisfying

$$
\left(0,0, \frac{1}{4 e}\right)\left(\begin{array}{c}
1 \\
x \\
x^{2}
\end{array}\right)= \begin{cases}0 & \text { if } x<e \\
x-e & \text { if } x \geqslant e\end{cases}
$$


Solutions: $x=0$ and $x=2 e$. The slack index $\sigma_{2}$ can also be used. Thus $M=\left(0,2 e, \sigma_{2}\right)$.

STEP 3 .

We look for $z_{1}, z_{2}$, $\xi$ satisfying

$$
\left(\begin{array}{ccc}
1 & 1 & 0 \\
0 & 2 e & 1 \\
0 & 4 e^{2} & 0
\end{array}\right) \quad\left(\begin{array}{l}
z_{1} \\
z_{2} \\
\xi
\end{array}\right)=\left(\begin{array}{l}
1 \\
\mu \\
\nu
\end{array}\right)
$$

Solution: $z_{1}=1-\frac{\nu}{4 e^{2}}, z_{2}=\frac{\nu}{4 e^{2}}, \xi=\mu-\frac{\nu}{2 e}$.

Here $F$ has the atoms $O$ and $2 e$ with masses

$$
m_{0}=1-\frac{\nu}{4 e^{2}}, m_{2 e}=\frac{\nu}{4 e^{2}} .
$$

STEP 4 .

$$
\int_{0}^{\omega}(x-e) d F_{x}=e \frac{\nu}{4 e^{2}}=\frac{\nu}{4 e}=\text { Value MIN in table } 2 .
$$

\subsection{Case $C_{22}^{\prime}$}

\section{STEP 2.}

We look for $x \in K$ satisfying

$$
\left(0,1-\frac{2 e \mu}{\nu}, \frac{e \mu^{2}}{v^{2}}\right)\left(\begin{array}{l}
1 \\
x \\
x^{2}
\end{array}\right)=\left\{\begin{array}{lr}
0 & \text { if } x<e \\
x-e & \text { if } x \geqslant e .
\end{array}\right.
$$

Solutions: $x=0, x=\frac{\nu}{\mu}$. Only the slack index $\sigma_{1}$ can also be used, but then we obtain the column $(1,0,0)^{\prime}$ already obtained for $x=0$. Since the use of the first slack index gives a defective distribution, we prefer to try

$$
M=\left(0, \frac{\nu}{\mu}, \frac{\nu}{\mu}\right) \text {. }
$$

STEP 3 .

We search for $z_{1}, z_{2}, z_{3}$ satisfying

$$
\left(\begin{array}{ccc}
1 & 1 & 1 \\
0 & \frac{\nu}{\mu} & \frac{\nu}{\mu} \\
0 & \frac{\nu^{2}}{\mu^{2}} & \frac{\nu^{2}}{\mu^{2}}
\end{array}\right)\left(\begin{array}{c}
z_{1} \\
z_{2} \\
z_{0}
\end{array}\right)=\left(\begin{array}{l}
1 \\
\mu \\
\nu
\end{array}\right) .
$$


The system is equivalent to: $z_{2}+z_{3}=\frac{\mu^{2}}{\nu}, z_{1}=1-\frac{\mu^{2}}{\nu}$. There is an indetermination in the choice of $z_{2}, z_{3}$, but the resulting distribution $F$ has anyway the two atoms o and $\nu / \mu$ with corresponding masses

$$
m_{0}=1-\frac{\mu^{2}}{\nu}, m_{v / \mu}=\frac{\mu^{2}}{\nu} .
$$

STEP 4 .

$$
\int_{0}^{\omega}(x-e) d F_{x}=\left(\frac{\nu}{\mu}-e\right) \frac{\mu^{2}}{\nu}=\mu\left(1-\frac{e \mu}{\nu}\right)=\text { Value MIN in table } 2 .
$$

$$
\text { 5. THE CASES } \nu \geqslant \mu \omega \text { AND } \mu^{2} \geqslant \nu
$$

These cases in which one constraint disappears are left as exercices for the reader. The answers can be found in table 3 .

TABLE 3

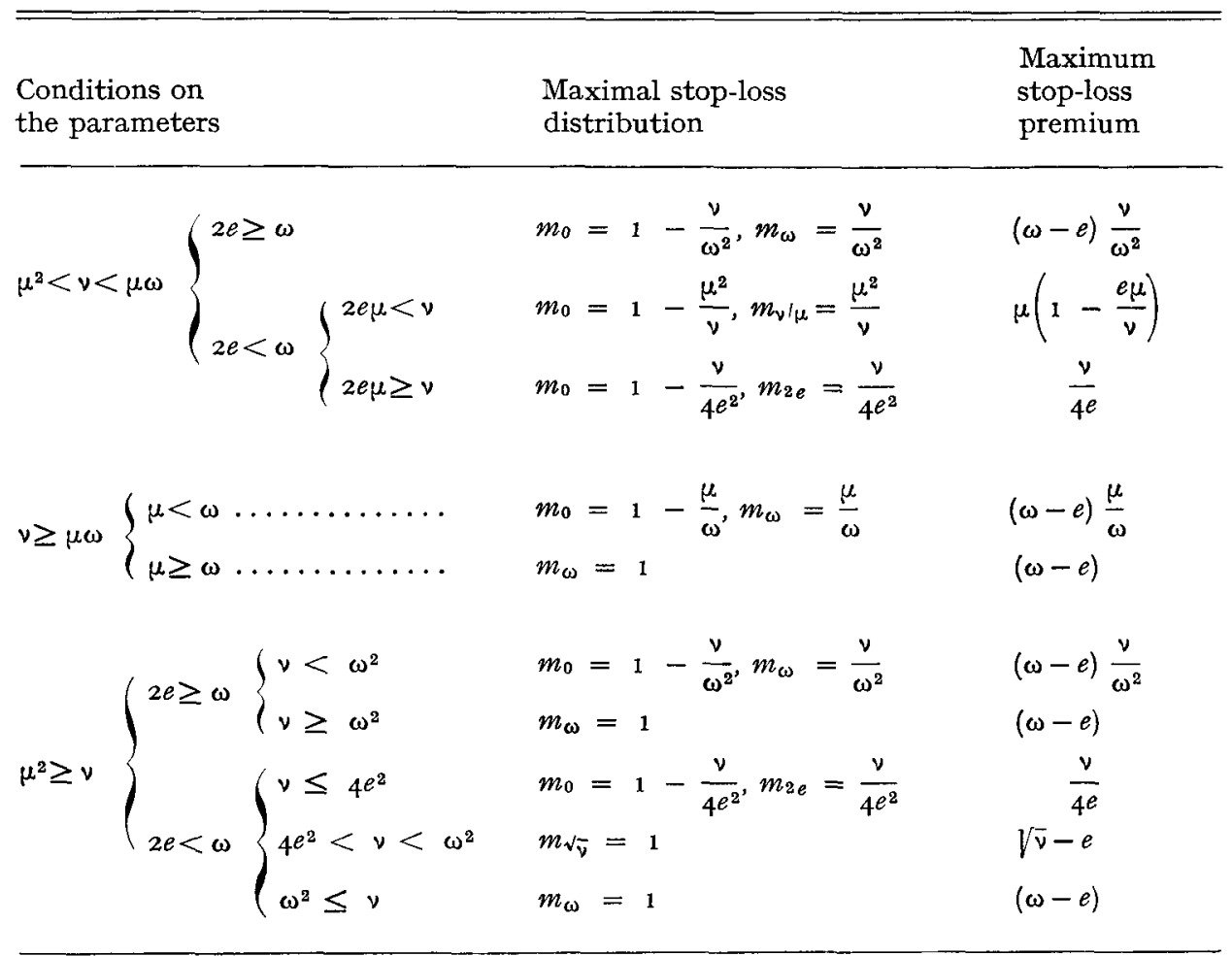




\section{SUMMARY}

A complete solution of the original problem in 2.1 is given in table 3 . It might seem surprising that we solved the modified problem in 2.2 and nevertheless always found a probability distribution. This is due to the fact, already observed in step 2 of the case $C_{22}^{\prime}$, that we never used the first slack index.

It is clear that several cases could be regrouped in table 3. For clearness, we leave it as it is. Indeed, given numerical values of the parameters, the table permits immediatly to situate the case to be used. Moreover, remind that the condition $\nu \geqslant \mu \omega$ amounts to the absence of the constraint on the second moment of $F$ and that the condition $\mu^{2} \geqslant \nu$ amounts to the absence of the constraint on the first moment of $F$. See the discussion in 2.4.

\section{REFERENCES}

MVSV = Mitteilungen der Vereinigung schweizerischer Versicherungsmathematiker. BühlmanN, H. (1974). Ein andere Beweis für die Stop-Loss-Ungleichung in der Arbeit Gagliardi/Straub. MVSV, 74.

De Vylder, F. (1978). Semi-continuous linear programming. MVSV, 78.

Gagliardi, B. and E. Straub. (1974). Eine obere Grenze für Stop-Loss-Prämien. MVSV, 74. 